\title{
A prospective study to evaluate the outcome of hepatitis b patients presenting with jaundice
}

\begin{abstract}
Background and study aims: Differentiating acute hepatitis B from reactivation of chronic hepatitis $\mathrm{B}(\mathrm{CHB})$ is essential. We evaluated consecutive patients presenting with prodrome and jaundice and detected to be HBsAg positive for the first time.

Patients and methods: All patients presenting with jaundice over a 6month period to the Kasturba Hospital of Infecious Disease (KHID), Mumbai between February and July 2015 with Hepatitis B surface antigen (HBsAg) positivity were included. Patients with previous knowledge of hepatitis B or cirrhosis, history of decompensation, significant alcohol or recent intake of hepatotoxic drugs were excluded. All patients were evaluated by a detailed history of clinical features and risk factors, examination, laboratory parameters and hepatitis B serological parameters. These patients were followed up for six months for determining their outcome.

Results: Of 1835 patients presenting with acute prodrome and jaundice, 65 patients were detected to be HBsAg positive. 51 patients completed the follow up of 6 months. 13 patients had acute hepatitis B and 38 had chronic hepatitis B (13 with superimposed acute hepatitis A or E over chronic hepatitis B and 25 patients with reactivation of hepatitis B). The hemogram and liver profile failed to differentiate acute from reactivation of hepatitis $\mathrm{B}$. Though median HBV load at presentation was higher in chronic hepatitis B it failed to be statistically significant between the groups (AUROC 0.6). $\mathrm{HBeAg}$ and Anti $\mathrm{HBe}$ failed to differentiate the two entities. IgM Anti $\mathrm{HBc}$ was more commonly positive in $\mathrm{AHB}$ and the sample/cutoff ratio was significantly higher in AHB with an AUROC of 0.81 . The cutoff of 11 reliably differentiates the two with a specificity of 100 and sensitivity of 57.1.
\end{abstract}

Conclusion: In patients presenting with jaundice and prodrome, nearly $75 \%$ are CHB. A Sample/cutoff ratio $>11$ for IgM Anti HBc can be used to identify patients with AHB.

Keywords: IgM Anti HBc, chronic hepatitis B, drug toxicity, bilirubin, transaminases, alkaline phosphatase

Abbreviation: $\mathrm{CHB}$, chronic hepatitis B; HBsAg, hepatitis B surface antigen; HPS, high pure system; ALF, acute liver failure; IgM, against core antigen; HBV, hepatitis B Virus

\section{Introduction}

India has over 40million HBV carriers and accounts for $10-15 \%$ of the entire pool of HBV carriers of the world. ${ }^{1}$ A meta-analysis of the prevalence of $\mathrm{HBV}$ had estimated that the point-prevalence of hepatitis B among nontribal and tribal populations was $3.07 \%$ and $11.85 \%$ respectively and the overall prevalence was $3.70 \% .{ }^{2}$ However, Lodha et al. did a systemic review of literature of prevalence of hepatitis B in India and concluded that the true prevalence of hepatitis $\mathrm{B}$ in India was $1-2 \% .{ }^{3,4}$ Spread of HBV infection in many South Asian countries is attributed to unsafe blood supply, reuse of contaminated syringes, lack of maternal screening to prevent perinatal transmission and delay in the introduction of hepatitis B vaccine. ${ }^{5}$ The predominant mode of transmission is horizontal rather than vertical in India. ${ }^{6}$

Patients with HBsAg positivity who present acutely with jaundice and prodromal symptoms may have acute HBV infection, superadded infections like hepatitis A, E or Non A to E virus, drug toxicity, or a reactivation of the HBV virus. This differentiation between the three groups is important as there is more than $95 \%$ chance of clearance of acute hepatitis B virus on conservative management. On the other hand, reactivation of chronic disease is an indication for starting treatment and may even lead to liver failure if not promptly treated. ${ }^{7-9}$ However,
Volume 10 Issue 6 - 2019

\author{
Pathik Parikh \\ Department of Hepatology, Liver Transplant, Zydus Hospitals, \\ India
}

Correspondence: Pathik Parikh, Department of Hepatology, Liver Transplant, Zydus Hospitals, India, Tel +9I-8652II9730, Email pathk269@gmail.com

Received: November 27, 2019 | Published: December 06, 2019 it may not be possible to differentiate them at the index presentation and require a follow up to determine the chronicity. Patients who fail to clear virus by six months are the ones with chronic disease or though less common, patients with acute hepatitis B becoming chronic. Both these groups require treatment. We prospectively evaluated patients presenting with acute jaundice and prodrome who were detected to be HBsAg positive with aim to characterize the outcome and hence determine the nature of hepatitis B infection.

\section{Patients and methods}

\section{Design}

This is a single center prospective study carried out at Kasturba Hospital of Infectious Disease (KHID), Mumbai a referral center for acute viral hepatitis between February and July 2015. An informed consent was taken from all patients. None of the authors declared any conflict of interest. An institutional ethics committee approval was obtained for the study.

\section{Inclusion and exclusion criteria}

Consecutive patients who were surface antigen positive and presenting with acute jaundice and prodrome were enrolled in the study. All patients with onset of illness within previous 8 weeks, with a bilirubin of greater than 2 and having an evidence of transaminitis of at least 5 times ULN were included. Patients with history of hepatitis $\mathrm{B}$ in the past or being a known case of any liver disease were excluded. 
Also all patients with age less than 15 years, older than 65 , presence of significant other comorbidities like alcohol use disorder or history of recent intake of hepatotoxic drugs were also excluded.

\section{Screening and evaluation}

A detailed history regarding the presenting complaints, possible source of infection, past and family history was taken. Patients were evaluated to rule out signs of chronic liver disease. Investigations in form of full blood count, bilirubin, transaminases, alkaline phosphatase, international standardized ratio of prothrombin (INR), albumin, IgM antibodies against hepatitis A (HAV-Ab) and E (HEV$\mathrm{Ab}$ ), hepatitis B surface antigen (HBsAg), antibody against hepatitis $\mathrm{C}(\mathrm{HCV}-\mathrm{Ab})$ and an ultrasound of the abdomen were carried out in all patients. In addition, hepatitis $\mathrm{B}$ viral quantification, antibody against surface antigen (Anti HBsAg), Hepatitis B e antigen (HBeAg), antibody against hepatitis $\mathrm{B}$ e antigen (Anti $\mathrm{HBe}$ ) and antibody against Hepatitis B core antigen (IgM-Anti $\mathrm{HBc}$ ) with sample to cutoff ratio were evaluated. These patients were followed up and bilirubin with transaminases and INR were repeated at days 7 and 14 and months 1 , 3 and 6 . HBsAg was repeated at 3 and 6 months.

We used $\operatorname{COBAS} \AA$ TaqMan ${ }^{\circledR}$ assay for quantification of viral load. The COBAS ${ }^{\circledR}$ TaqMan ${ }^{\circledR}$ HBV Test For Use With The High Pure System (HPS) is an in vitro nucleic acid amplification test for the quantitation of Hepatitis B Virus (HBV) DNA in human serum or plasma, using the High Pure System Viral Nucleic Acid Kit for manual specimen preparation and the COBAS ${ }^{\circledR}$ TaqMan ${ }^{\circledR} 48$ Analyzer for automated amplification and detection. Sample-Cutoff ratio for Anti $\mathrm{HBc}$ IgM was evaluated by ARCHITECT, ANTI HBC-IgM 6c33 kits from Abbott and the information was processed by ARCHITECT iSystem software. Any value $\geq 1$ is given as a positive result. The system is referenced against the Paul Ehrlich Institute, Largen Germany (HBc Referenzserum IgM 84). Patients were evaluated for anti $\mathrm{HBc}$ IgM within 7 days of the day of presentation.

\section{Treatment criteria}

All patients with development of signs of liver failure in the form of ascites or encephalopathy during anytime from admission to six months were started on treatment. Besides, if one month after the onset of jaundice, the bilirubin remained higher than $10 \mathrm{mg} / \mathrm{dl}$ or INR $>1.5$ (severe acute hepatitis B as per AASLD guidelines) ${ }^{10}$ then the patient was started on treatment. Finally, if HBsAg remained positive at 6 months, treatment was started as it was most likely due to reactivation of hepatitis $B$ if $A L T \geq 2 U L N, H B V \geq 2000$. All patients whom treatment was considered were treated with tenofovir $300 \mathrm{mg}$ once a day.

\section{Statistics}

SPSS 20 was used for calculations and $p$ value less than 0.05 was considered as significant. ANOVA and Student's t test were utilized for calculating the significance between groups with continuous data while Fiscer's exact test was used for calculating the significance between discrete data. The comparisons are made initially between three groups, 1) acute hepatitis B, 2) Chronic hepatitis B with acute superimposed Hepatitis A or E and 3) Reactivation of chronic hepatitis B. Secondly the comparisons are made between the chronic hepatitis B group as a whole ( $2 \& 3$ combined) with Acute hepatitis B. ROC curve was plotted for IgM Anti HBc to determine the cutoff for acute hepatitis B.

\section{Results}

In the period of six months, a total of 1835 patients presented to the hospital with acute prodrome and jaundice. A total of $65(3.5 \%)$ consecutive patients were detected to be hepatitis B (HBsAg Positive) during the period of study were included. Fifty one who had at least 6 months follow up have been analyzed for their outcome at six months. Figure 1 depicts the flow chart of patients in the study.

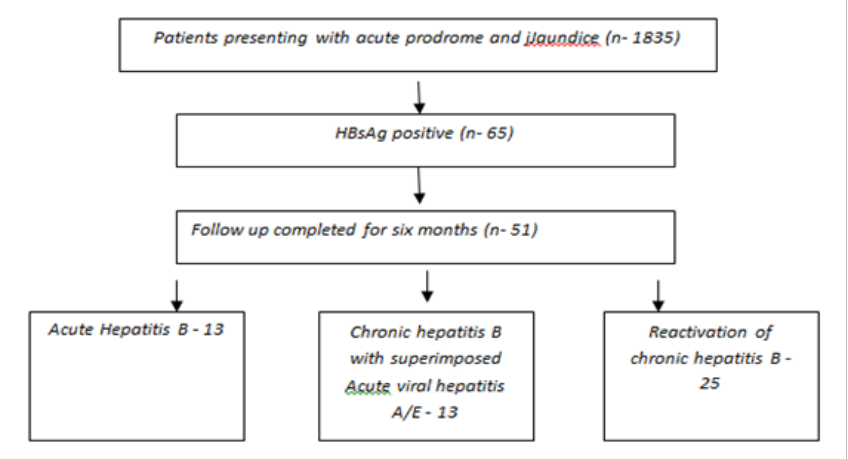

Figure I The flowchart of patients included in the study. I 4 patients who did not complete a six month followup were excluded from the study.

\section{Demography}

The mean age of presentation was 34.5 years with $70.5 \%$ being males, majority (76.5\%) were residents of Mumbai city. When we compared the demographic data of patients who turned out to be acute hepatitis B, chronic hepatitis B with superimposed acute hepatitis A or $\mathrm{E}$ and Reactivation of chronic hepatitis $\mathrm{B}$, there was no difference in age, gender predominance or the location from where they belonged. Though, patients who developed a superimposed acute hepatitis A or E appeared to be younger, the difference was not significant. Similarly, when chronic hepatitis B was taken as a whole group, even then there was no statistical difference in demographic features of the acute and chronic groups (Tables $1 \& 2$ ).

\section{Clinical presentation}

At presentation, the mean duration of symptoms were $21.2 \pm 2.2$ days with mean duration of jaundice being 13.4 \pm 1.4 days (Mean \pm standard error of mean). Gastrointestinal symptoms (Anorexia, nausea and vomiting, abdominal discomfort/pain) were most common symptoms at presentation, after jaundice, being seen in $65 \%$ patients while other symptoms were fatigue (65\%), malaise $(65 \%)$, fever $(51 \%)$, weight loss $(22 \%)$ and itching in (11\%). None of the patients with acute hepatitis B had itching as a symptom. However, the symptoms at presentation including itching failed to predict the outcome as there was no statistical difference between acute hepatitis B, superimposed hepatitis $\mathrm{A} / \mathrm{E}$ and reactivation of hepatitis $\mathrm{B}$. Further, there was no statistical significant difference between acute and chronic hepatitis B groups including reactivation a superimposed group. (Table 1 and Table 2) None of the patients in either group had any symptom or sign suggestive of chronic liver disease at presentation (ascites, pedal edema, encephalopathy, gastrointestinal bleed, spiders). However one patient developed ascites after 25 days of admission and was found to have chronic hepatitis B. His ultrasound showed no evidence of cirrhosis at presentation. 
Table I It shows the demography, clinical features, risk factors and investigations in patients presenting with jaundice and turning out to be Acute Hepatitis B, Chronic Hepatitis B with superimposed Acute Hepatitis A/E, and Recativation of chronic Hepatitis B

\begin{tabular}{|c|c|c|c|c|}
\hline & $\begin{array}{l}\text { Acute Hepatitis } \\
\text { B }(n=13)\end{array}$ & $\begin{array}{l}\text { Superimposed AVH } \\
A / E \text { on } C H B(n=13)\end{array}$ & $\begin{array}{l}\text { Reactivation of } \\
\text { CHB }(n=25)\end{array}$ & $P$ value \\
\hline Age & $34.7 \pm 2.3$ & $28.3 \pm 3.6$ & $36.2 \pm 2.2$ & 0.12 \\
\hline Males & $76.9 \%$ & $69 \%$ & $68 \%$ & 0.30 \\
\hline Origin from Within Mumbai & $76.9 \%$ & $76.9 \%$ & $76 \%$ & 0.98 \\
\hline Duration of Symptoms (days $\pm S E$ ) & $16.6 \pm 4.8$ & $19.4 \pm 4.4$ & $20.6 \pm 3.0$ & 0.98 \\
\hline Duration of Jaundice (days \pm SE) & $10.1 \pm 2.1$ & $12.0 \pm 2.9$ & $14.0 \pm 2.0$ & 0.72 \\
\hline History of Jaundice & $0(0)$ & $8 \%(n=1)$ & $12 \%(n=3)$ & 0.42 \\
\hline History of Surgery & $0(0)$ & $8 \%(n=I)$ & $28 \%(n=7)$ & 0.05 \\
\hline History of dental procedures & $15 \%(n=2)$ & $38 \%(n=5)$ & $28 \%(n=7)$ & 0.41 \\
\hline History of IM Injections & $61 \%(n=8)$ & $61 \%(n=8)$ & $52 \%(n=13)$ & 0.79 \\
\hline Screening for HBV in family $(\mathrm{n})$ & 8 & 6 & 20 & - \\
\hline $\begin{array}{l}\text { Number of positives detected on } \\
\text { screening (\%) }\end{array}$ & 0 & $0(0)$ & 2 & - \\
\hline Unsafe sexual practice & $31 \%(n=4)$ & $15 \%(n=2)$ & $12 \%(n=3)$ & 0.34 \\
\hline Tattooing & $31 \%(n=4)$ & $0(0)$ & $16 \%(N=4)$ & 0.09 \\
\hline Piercing & $0(0)$ & $0(0)$ & $8 \%(N=2)$ & 0.33 \\
\hline Shaving in unhygienic place & $38 \%(n=5)$ & $31 \%(n=4)$ & $24 \%(n=6)$ & 0.64 \\
\hline Haemoglobin & $12.3 \pm 0.4$ & $13.6 \pm 0.5$ & $12.9 \pm 0.2$ & $0.04 *$ \\
\hline Total leukocyte count & $11260 \pm 1294$ & $9130 \pm 646$ & $9090 \pm 631$ & 1.00 \\
\hline Platelets & $1.61 \pm 2.0$ & $2.24 \pm 2.8$ & $2.06 \pm 1.6$ & 0.37 \\
\hline Bilirubin & $20.3 \pm 2.7$ & $14.5 \pm 2.5$ & $17.7 \pm 1.5$ & 0.31 \\
\hline Direct bilirubin & $9.7 \pm 1.3$ & $7.8 \pm 1.2$ & $8.8 \pm 3.7$ & 0.63 \\
\hline AST & $860 \pm 208$ & $823 \pm 192$ & $768 \pm 116$ & 0.96 \\
\hline ALT & $891 \pm 140$ & $830 \pm|7|$ & $859 \pm 120$ & 0.99 \\
\hline ALP & $124 \pm 11$ & $123 \pm 10.5$ & $163 \pm 18$ & 0.19 \\
\hline INR & $1.2 \pm 0.08$ & $1.2 \pm 0.07$ & $1.2 \pm 0.04$ & 0.73 \\
\hline Proteins & $7.3 \pm 0.1$ & $7.1 \pm 0.2$ & $7.4 \pm 0.1$ & 0.49 \\
\hline Albumin & $3.5 \pm 0.1$ & $3.9 \pm 0.1$ & $3.5 \pm 0.07$ & $0.02 * *$ \\
\hline HAV IgM positive & $8 \%(n=1)$ & $23 \%(n=3)$ & $4 \%(n=1)$ & 0.164 \\
\hline HEV IgM positive & $0(0)$ & $77 \%(n=10)$ & 0 & $<0.00001$ \\
\hline HCV positive & $0(0)$ & 0 & 0 & - \\
\hline Igm Anti HBc & $100 \%(n=13)$ & $0(0)$ & $76 \%(n=19)$ & $<0.00001$ \\
\hline $\begin{array}{l}\text { Median titres of IgM AntiHbc (S/ } \\
\text { CO) }\end{array}$ & 13.143 .4 & & 6.40 .9 & $0.02 * * *$ \\
\hline
\end{tabular}




\begin{tabular}{lllll}
\hline & $\begin{array}{l}\text { Acute Hepatitis } \\
\text { B }(\mathbf{n}=13)\end{array}$ & $\begin{array}{l}\text { Superimposed AVH } \\
\text { A/E on CHB }(\mathbf{n}=13)\end{array}$ & $\begin{array}{l}\text { Reactivation of } \\
\text { CHB }(\mathbf{n}=25)\end{array}$ & P value \\
\hline Anti HBe & $85 \%(n=1)$ & $92 \%(n=12)$ & $76 \%(n=19)$ & 0.44 \\
HBeAg & $23 \%(n=3)$ & $8 \%(n=1)$ & $12 \%(n=3)$ & 0.49 \\
HBV Load at diagnosis (Mean IU/ & 10597 & 8529675 & 134199936 & 0.62 \\
ml) & $38.4 \%(n=5)$ & $30.7 \%(n=4)$ & $20 \%(n=5)$ & 0.45 \\
HBV undetectable at presentation & $8 \%(n=1)$ & $23 \%(n=3)$ & $28 \%(n=7)$ & 0.34 \\
Bilirubin > I0 mg/dl at 28 days & $8 \%(n=1)$ & $100 \%(n=13)$ & $100 \%(n=25)$ & $<0.00001$ \\
HBs positivity at 3 months & &
\end{tabular}

ANOVA is used to determine significance for continuous data. Fisher's exact test is used for discrete data for determination of significance. $P$ value $<0.05$ is considered significant.

*Significance was detected only between Acute and Superimposed groups.

**Significance was detected only between reactivation and superimposed groups.

***Significance was detected between Acute and Reactivation group

None of the patients had history of needle stick injury or intravenous drug use or having history of hepatitis B in family. None were vaccinated and none had signs and symptoms of chronicity

Table 2 It shows the demography, clinical features, risk factors and investigations in patients presenting with jaundice and turning out to be Acute Hepatitis B, Chronic Hepatitis B

\begin{tabular}{|c|c|c|c|}
\hline & $\begin{array}{l}\text { Acute Hepatitis B } \\
(n=13)\end{array}$ & CHB $(n=38)$ & $P$ value \\
\hline Age & 34.72 .3 & 33.832 .2 & 0.82 \\
\hline Gender (Males) & $76.9 \%$ & $68.4 \%$ & \\
\hline Origin from Within Mumbai & $76.9 \%$ & $77 \%$ & \\
\hline Duration of Symptoms & 16.64 .8 & 19.52 .2 & 0.74 \\
\hline Duration of Jaundice & 10.12 .1 & 13.01 .6 & 0.84 \\
\hline History of Jaundice & $0(0)$ & $10 \%(n=4)$ & 0.56 \\
\hline History of Surgery & $0(0)$ & $21 \%(n=8)$ & 0.09 \\
\hline History of dental procedures & $15 \%(n=2)$ & $31 \%(n=\mid 2)$ & 0.47 \\
\hline History of IM Injections & $61 \%(n=8)$ & $55 \%(n=21)$ & 0.75 \\
\hline Screening for HBV in family & 8 & 26 & - \\
\hline Number of positives detected on screeing & 0 & $5 \%(n=2)$ & 1.0 \\
\hline Unsafe sexual practice & $31 \%(n=4)$ & $13 \%(n=5)$ & 0.20 \\
\hline Tatooing & $31 \%(n=4)$ & $10 \%(n=4)$ & 0.17 \\
\hline Piercing & $0(0)$ & $5 \%(n=2)$ & 1.0 \\
\hline Shaving in unhygienic place & $38 \%(n=5)$ & $26 \%(n=10)$ & 0.48 \\
\hline Signs or symptoms of chronicity & $0(0)$ & $3 \%(n=1)$ & 1.0 \\
\hline Hemoglobin & 12.30 .4 & 13.30 .2 & 0.01 \\
\hline Total leucocyte count & $1|260| 294$ & 8953349 & 1.00 \\
\hline Platelets & 1.612 .0 & 2.171 .4 & 0.14 \\
\hline Bilirubin & 20.32 .7 & 16.11 .3 & 0.20 \\
\hline Direct bilirubin & 9.71 .3 & 8.30 .6 & 0.40 \\
\hline AST & 860208 & 793100 & 0.88 \\
\hline
\end{tabular}




\begin{tabular}{|c|c|c|c|}
\hline & $\begin{array}{l}\text { Acute Hepatitis B } \\
(n=\mid 3)\end{array}$ & CHB $(n=38)$ & $P$ value \\
\hline ALT & 891140 & 86497 & 0.12 \\
\hline ALP & 124|| & 14913 & 0.44 \\
\hline INR & 1.20 .08 & 1.20 .03 & 0.72 \\
\hline Proteins & 7.30 .1 & 7.30 .11 & 1.0 \\
\hline Albumin & 3.50 .1 & 3.60 .07 & 0.92 \\
\hline HAV IgM positive & $8 \%(n=I)$ & $10 \%(n=4)$ & 1.0 \\
\hline HEV IgM positive & $0(0)$ & $26 \%(n=10)$ & 0.04 \\
\hline HCV positive & $0(0)$ & 0 & - \\
\hline $\operatorname{lgm}$ Anti $\mathrm{HbC}$ & $100 \%(n=13)$ & $50 \%(n=19)$ & 0.0008 \\
\hline Median titres of IgMAntiHbc (S/CO) & 13.143 .4 & 6.40 .9 & 0.02 \\
\hline Anti HBS & $38 \%(n=5)$ & $7 \%(n=3)$ & 0.01 \\
\hline Anti $\mathrm{HBe}$ & $85 \%(n=11)$ & $81 \%(n=31)$ & 1.0 \\
\hline $\mathrm{HBeAg}$ & $23 \%(n=3)$ & $10 \%(n=4)$ & 0.35 \\
\hline HBV Load at diagnosis (Mean IU/ml) & 10597 & 6623938 & 0.35 \\
\hline HBV Undetectable at presentation & $38.4 \%(n=5)$ & $23.6 \%(n=9)$ & 0.30 \\
\hline Bilirubin $>10 \mathrm{mg} / \mathrm{dl}$ at 28 days & $8 \%(n=1)$ & $26 \%(n=10)$ & 0.24 \\
\hline HBs positivity at 3 months & $8 \%(n=1)$ & $100 \%(n=38)$ & $<0.0001$ \\
\hline
\end{tabular}

Students $\mathrm{t}$ test is used to determine significance for continuous data. Fisher's exact test is used for discrete data for determination of significance. $\mathrm{P}$ value $<$ 0.05 is considered significant

\section{Risk factors for hepatitis B}

The three most common risk factors elucidated from the patients were history of recurrent intramuscular injections (more than 2 per year) in 58\% patients, shaving outside with questionable sterility of blades in 30\% patients and history of some dental procedures (not including scaling) in 28\% patients. The other risk factors were history of unsafe sexual practices (unprotected intercourse in a nonmonogamous relationship) (17\%), Tattooing (16\%), history of previous surgery (16\%), previous history of jaundice $(8 \%)$ and piercing (4\%). A patient gave history of zangir-zani (beating oneself with chains). When we compared the three groups there was no difference in the number of risk factors between the three groups as well as between acute and chronic hepatitis. Of note, none of the patients with acute hepatitis B gave history of any surgery in past, and the difference was close to being significant $(\mathrm{p}=0.05)$. We did not find history of needle stick injury, blood transfusion or intravenous drug use in any patient.

When inquired about hepatitis B vaccination or liver disease in family, none of the patients in either group gave a positive history. When advised, 12 (23\%) patients got their family members evaluated. In a total of 34 family members who were evaluated, we picked up $2(5 \%)$ positive members (Siblings). Both these patients with family members positive, turned out to be chronic hepatitis $\mathrm{B}$ reactivation (Tables 1 \& 2).

\section{Laboratory investigations}

The difference between the acute and chronic hepatitis B was significant in the levels of hemoglobin, though when comparison was made between acute, superimposed hepatitis $\mathrm{A} / \mathrm{E}$ and chronic group, it was found significantly different between acute hepatitis B and Superimposed Hepatitis A/E group. Similarly, in the serum albumin levels, there was no statistical significant difference between acute and chronic group but in the chronic group itself, it was lower in patients with reactivation of chronic hepatitis $\mathrm{B}$ as compared to superimposed group. There is no difference in bilirubin levels at the end of 28 days between each group and prolonged jaundice may not be able to pick up chronic hepatitis B. Figure 2A, 2B and 2C denotes the progression of bilirubin, ALT and AST among patients with acute hepatitis B, Superimposed hepatitis A/E on CHB, and chronic hepatitis $\mathrm{B}$ reactivation.

The likelihood of chronicity is significantly higher for being a chronic hepatitis when antibodies against hepatitis $\mathrm{E}$ is detected $(\mathrm{p}=0.04)$ (Tables $1 \& 2)$. All patients underwent an ultrasound of abdomen and it did not show evidence of chronic liver disease or portal hypertension in any patient.

\section{Hepatitis B serology}

Antibody against core antigen (IgM) is statistically more common in acute hepatitis B as compared to chronic hepatitis B. All patients with acute hepatitis B had positive IgM AntiHbc. However, 76\% patients with reactivation had positive IgM AntiHBc. The sample/ cutoff ratio in acute hepatitis $B$ was significantly higher in acute hepatitis B as compared to chronic hepatitis B with reactivation. The ROC for IgM Anti HBc to detect acute hepatitis B is 0.810 (95\% CI, 0.587-0.943). Figure 3 A shows the ROC curve. 


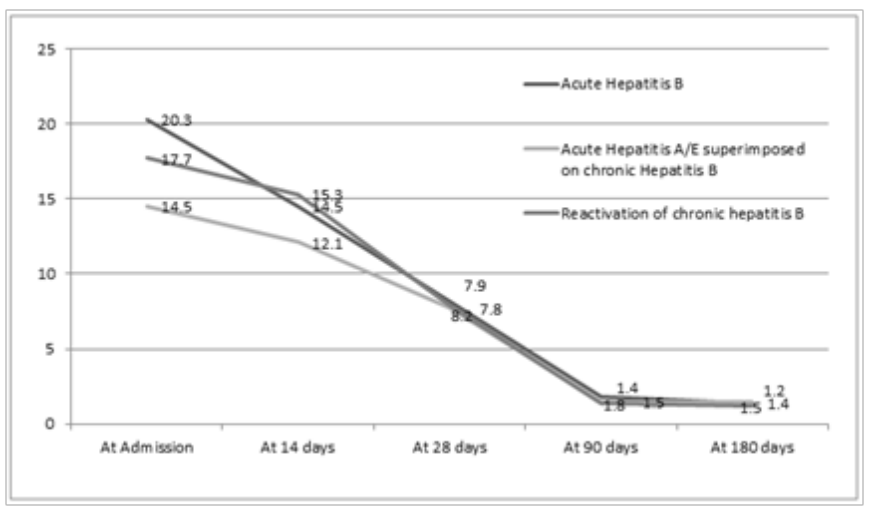

Figure 2A The figure shows the bilirubin levels in patients with Acute hepatitis B, Chronic Hepatitis B with Superimposed acute hepatitis A/E and Reactivation of chronic hepatitis $B ;(P>0.05)$.

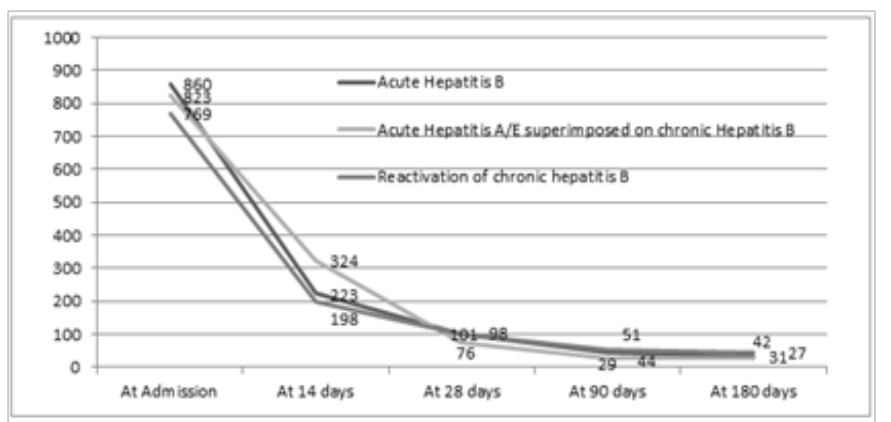

Figure 2B The figure shows the ALT levels in patients with Acute hepatitis B, Chronic Hepatitis B with Superimposed Acute hepatitis A/E and Reactivation of chronic hepatitis $B ;(P>0.05)$.

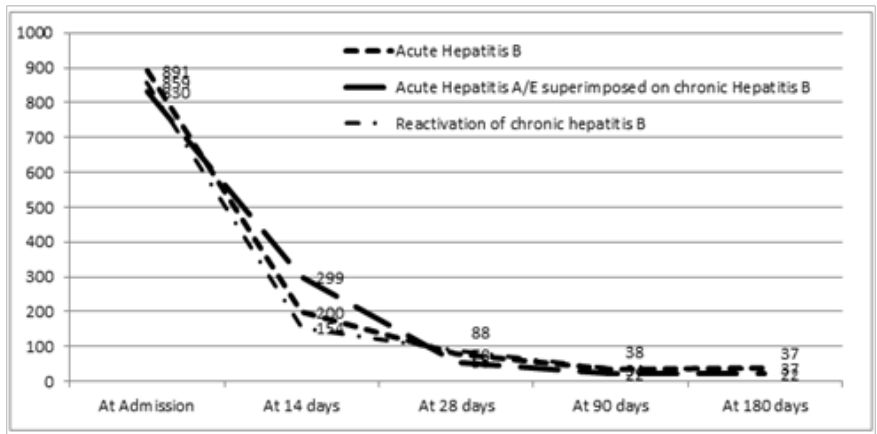

Figure 2C The figure shows the AST levels in patients with Acute hepatitis B, Chronic Hepatitis B with Superimposed acute hepatitis A/E and Reactivation of chronic hepatitis $B ;(P>0.05)$.

Envelope antigen and antibody against envelope antigen fails to differentiate acute from chronic hepatitis B with or without reactivation. Though, mean HBV load at presentation is higher in chronic hepatitis B it failed to be statistically significant between the groups. The AUROC for HBV viral quantification to pick differentiate acute from chronic hepatitis $\mathrm{B}$ at the time of presentation is 0.624 (Figure 3B). When these patients were followed up for 6 months, patients with $\mathrm{HBsAg}$ positivity at three months were more likely to be chronic. Only one patient of 13 with acute hepatitis B had positive HBsAg at 3 months who subsequently became negative by six months.

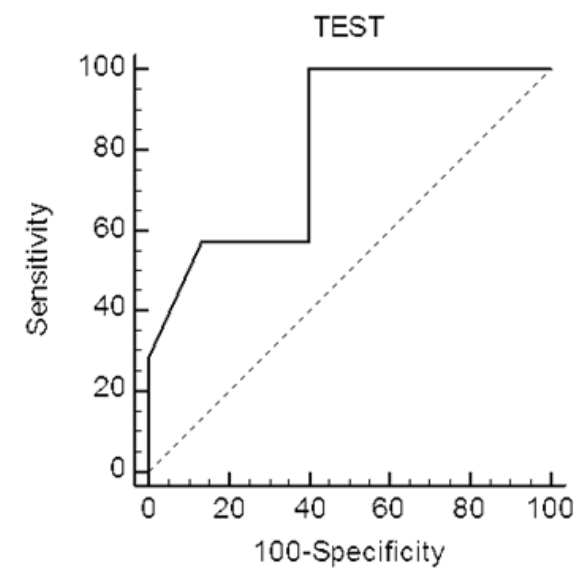

Figure 3A It shows the ROC curve for IgMAnti $\mathrm{HBc}$ levels to detect acute Hepatitis B.

\section{ROC Curve}

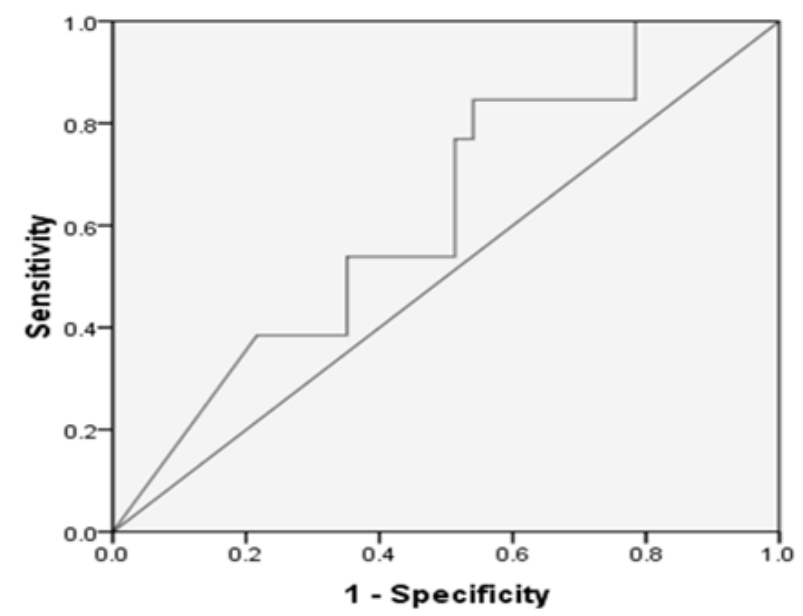

Figure 3B It shows the ROC curve for HBV quantification levels to detect acute Hepatitis B.

\section{Discussion}

Hepatitis B transmission in India is mainly vertical or horizontal and mainly during childhood rather than in adulthood. Majority are not aware of the status of hepatitis B until they get tested for some reason. In this study, patients presenting like acute hepatitis with prodrome and jaundice, unaware of their hepatitis B status or vaccination in the past are prospectively studied. Demography, clinical presentation, risk factors or even routine laboratory features fail to differentiate acute hepatitis B from chronic hepatitis B. The differentiation between these two conditions is important because in cases of acute hepatitis, except for very severe ones, antiviral therapy is not recommended, whereas this may be indicated in majority of patients with chronic hepatitis. To the best of our knowledge this is a first study that prospectively evaluated HBsAg patients presenting with jaundice. A retrospective analysis published from Delhi addressed the similar issue and did not find any difference in the groups in relation to clinical and biochemical features. ${ }^{11}$ 


\section{Role of HBV DNA in the differential diagnosis}

It has been shown that HBV DNA levels may help differentiate an acute infection from an exacerbation of chronic infection, which requires therapy. In the study from Delhi, low levels of HBV DNA $(<141500$ copies $/ \mathrm{mL})$ were found in about $96 \%$ of patients with acute infection, as opposed to $13 \%$ in those with exacerbation of chronic hepatitis. Higher HBV DNA levels ( $>10^{5}$ copies per $\mathrm{ml}$ ) were found in $87 \%$ of patients with chronic infection compared to only $4 \%$ in those with acute infection ${ }^{11}$. The sensitivity and specificity of low levels of HBV DNA for identifying an acute infection are $96 \%$ and $86.6 \%$, respectively. ${ }^{11}$ Han et al. ${ }^{12}$ found that not only was an undetectable level of serum HBVDNA was more often seen in patients with acute hepatitis-B than in reactivation of chronic hepatitis B patients $(22.1 \%$ vs. $6.2 \%, \mathrm{P}<0.001$ ), but also the average concentration was lower in acute than in reactivation patients among those with positive serum HBVDNA (4.53 vs. $6.59 \log 10$ copies $/ \mathrm{mL}$ ). Dao et al. ${ }^{13}$ found that in HBV related acute liver failure (ALF), the viral loads at admission were lower in patients with acute hepatitis B than in reactivation of chronic (3.9 log vs. $5.2 \mathrm{log})$. There was a significant overlap in admission viral loads between acute and reactivation related ALF. In the patients with reactivation, those who were immunosuppressed had higher viral loads while those who were non-immunosuppressed had viral loads similar to acute hepatitis B. Our study shows contradictory findings to the previous study from Delhi for utilising viral load. Only $16 \%$ patients with reactivation of chronic hepatitis B showed a viral load greater than $10^{6}$ copies per $\mathrm{ml}$ and $20 \%$ showed a viral load that was less than 100 copies per $\mathrm{ml}$. There was insignificant difference between the viral loads in acute viral hepatitis group and the reactivation of chronic hepatitis B group. It could be explained by the fact that Immune activation during reactivation of hepatitis B may bring down the viral load considerably and by the time patient presents with jaundice, the viral load may have been significantly reduced. However, a noteworthy point in our study was that none of the patients with acute hepatitis B had a viral load greater than $10^{6}$. As less number of patients had viral load greater than $10^{6}$ copies per $\mathrm{ml}$, the difference was not statistically significant. It is likely that if present, a viral load greater than $10^{6}$ copies per $\mathrm{ml}$ has a good predictive value in excluding acute hepatitis B.

\section{Role of HBeAg in the differential diagnosis}

The presence of HBeAg is usually associated with high levels of HBV DNA in serum. Seroconversion from $\mathrm{HBeAg}$ carrier to anti-HBe antibodies occurs early in patients with acute infection, prior to $\mathrm{HBsAg}$ to anti-HBs seroconversion. ${ }^{14}$ However, $\mathrm{HBeAg}$ seroconversion may be delayed for years in patients with chronic $\mathrm{HBV}$ infection. In the present study, $\mathrm{HBeAg}$ and Anti $\mathrm{HBe}$ failed to differentiate acute from chronic infection as was noted in the study from Delhi. In acute infection if the patient presents early, may have $\mathrm{HBeAg}$ positivity which gets seroconverted if presented late during the illness. Similarly, in chronic hepatitis B with reactivation, there may be seroconversion. These markers do not help differentiate the two conditions.

\section{Role of IgM Anti-HBcAg in the differential diagnosis}

The viral core antigen is expressed within the infected hepatic cells and cannot be detected in serum. Its corresponding antibody, on the other hand, can be detected in serum at different phases of infection. $\operatorname{IgM}$ anti-HBc is a single serum marker of $\mathrm{HBV}$ infection within the period between the disappearance of HBsAg and the appearance of anti-HB antibodies. Identification of $\mathrm{IgM}$ anti-HBc is considered diagnostic for the acute phase of infection, but it has been reported that it can remain present in serum for two years from the initial infection. ${ }^{11,15}$ Moreover, the titre of IgM anti-HBc can also rise and become detectable in exacerbations of chronic hepatitis Kumar et al. ${ }^{11}$ demonstrated that high titres of IgM anti-HBc are more common in patients with acute infection, and titres above 1:1000 can be seen in up to $80 \%$ of these patients. In about $70 \%$ of patients with chronic hepatitis, $\operatorname{IgM}$ anti-HBc titres were lower than 1:1000 or negative. However, differentiation between these two conditions by measuring the titres did not prove to have high sensitivity, specificity, NPV or PPV. Another assay has been proposed in literature that enables the standardization between different laboratories, i.e. the sample/cut-off ratio $(\mathrm{S} / \mathrm{CO})$. A ratio $>10$ indicates acute infection whereas a ratio $<10$ indicates chronic infection. An S/CO $>10$ had a sensitivity and NPV of $100 \%$, a specificity of $99 \%$ and PPV of $99.3 \%$ for diagnosing an acute hepatitis B. ${ }^{16}$ Rodella et al. ${ }^{16}$ by quantitative assay found $\operatorname{IgM}$ anti-HBc antibodies were positive in $32.85 \%$ in patients with chronic hepatitis B though at lower levels than AVH-B. They found that average sample/cutoff (S/CO) value of $>10$ for IgM anti-HBc identified $\mathrm{AVH}-\mathrm{B}$ while in $\mathrm{CHB}-\mathrm{AE}$ the $\mathrm{S} / \mathrm{CO}$ values were $<10$. Dao et al. $^{13}$ found that, the IgM anti-HBc levels by quantitative immunoassay were much higher in liver failure related to $\mathrm{CHB}-\mathrm{AE}$ than in liver failure related to $\mathrm{AVH}-\mathrm{B}$ with a signal-to-noise $(\mathrm{S} / \mathrm{N})$ ratio of 5.0 identifying $96 \%$ of AVH-B. Similar findings were observed in our study where a ratio greater than 10 was highly suggestive of acute infection. So we recommend utilizing the ratio for diagnosing acute hepatitis B.

\section{Role of other viral serology in the differential diagnosis}

The likelihood of getting two acute viral infections simultaneously is minimal. So in a patient with HBsAg positivity and getting IgM HAV or IgM HEV positive, it is likely to be a chronic hepatitis B with superimposed acute viral hepatitis. In our study HEV positivity was seen in chronic hepatitis B only, though in view of less number of patients the positivity of IgM HAV did not show significant difference between two groups.

\section{Risk factors for hepatitis B in our population}

We evaluated all patients by questionnaire about the risk factors. The importance of recurrent intramuscular injections (58\%), shaving outside with no knowledge about the use of new blades (30\%) and dental procedures $(28 \%)$ needs to be highlighted as a risk factor for hepatitis $\mathrm{B}$. The evidence of intramuscular injection can also be provided with a recent outbreak of acute severe hepatitis B with acute liver failure in Gujarat in 2008. ${ }^{17}$ We describe for the first time in literature a patient of hepatitis B transmission possibly caused by zangir-zani. This is a ritual associated with Islam, which takes place in Muharram, the first month of the Islamic calendar. Expressions of grief such as sinezani (beating the chest), zangir-zani (beating oneself with chains), and tage-zani or qama-zani -also known as tatbir (hitting oneself with swords or knives)- emerged as common features of the proliferating mourning-processions. Needle stick injury, sexual transmission and IV drug use which are common mode of transmission in the West did not form a major risk factor in our population. This emphasizes on the fact that a different strategy from the West is required in India for prevention of spread of hepatitis B. The limitation of our study includes non-measurement of serum HBsAg levels (Quantitative) and not obtaining histological or noninvasive assessment of chronicity. Nevertheless, the prospective nature of the study and six month follow up are the strong points in the study. 


\section{Conclusion}

To conclude, HBsAg positivity was seen in $3.5 \%$ of all our patients presenting with features suggestive of acute viral hepatitis. Seventy five percent of these are chronic and fifty percent of the chronic are actually reactivation of chronic hepatitis B. IgM Anti $\mathrm{HBc}$ tires play an important role in differentiating acute hepatitis $B$ from reactivation. A sample/cutoff ratio of $>10$ can be utilized for identifying acute hepatitis $\mathrm{b}$. A viral load usually is below $10^{6}$ copies per $\mathrm{ml}$ in almost all cases at presentation and may not be helpful to differentiate acute from chronic hepatitis B. Recurrent Intramuscular injection, unhygienic shaving and history of dental extraction are prominent risk factors for hepatitis B in Indian population.

\section{Acknowledgments}

None.

\section{Conflicts of interest}

Author declare sheer are no conflicts of interest.

\section{References}

1. Puri P. Tackling the Hepatitis B Disease Burden in India. J Clin Exp Hepatol. 2014;4(4):312-319.

2. Batham A, Gupta MA, Rastogi P, et al. Calculating prevalence of hepatitis $\mathrm{B}$ in India: using population weights to look for publication bias in conventional meta-analysis. Indian J Pediatr. 2009;76(12):1247-1257.

3. Lodha R, Jain Y, Anand K, et al. Hepatitis B in India. A review of disease epidemiology. Indian Pediatr. 2000;38(4):349-371.

4. Patil S, Rao RS, Agarwal A. Awareness and risk perception of hepatitis B infection among auxiliary healthcare workers. J Int Soc Prev Community Dent. 2013;3(2):67-71.

5. Tandon BN, Acharya SK, Tandon A. Epidemiology of hepatitis B virus infection in India. Gut. 1996;38(Suppl 2):S56-S59.

6. Gupta S, Gupta R, Joshi YK, et al. Role of horizontal transmission in hepatitis B virus spread among household contacts in North India. Intervirology. 2008;51(1):7-13.
7. Puri P. Acute Exacerbation of Chronic Hepatitis B: The Dilemma of Differentiation from Acute Viral Hepatitis B. J Clin Exp Hepatol. 2013;3(4):301-312.

8. Kumar M, Jain S, Sharma BC, et al. Differentiating acute hepatitis B from first episode of symptomatic exacerbation of chronic hepatitis B. Dig Dis Sci. 2006;51(3):594-599.

9. Pan CQ, Zhang JX. Natural History and Clinical Consequences of Hepatitis B Virus Infection. Int J Med Sci. 2005;2(1):36-40.

10. Lok AS, McMohan BJ. Chronic hepatitis B: update of recommendations. Hepatology. 2004;39(3):857-861.

11. Kumar M, Jain S, Sharma BC, et al. Differentiating acute hepatitis B from the first episode of symptomatic exacerbation of chronic hepatitis B. Dig Dis Sci. 2006;51(3):594-599.

12. Han Y, Tang Q, Zhu W, et al. Clinical, biochemical and serological profiles of, and differential diagnosis between, patients with acute hepatitis B and chronic hepatitis B with acute flare. $J$ Gastroenterol Hepatol. 2008;23(11):1728-1733.

13. Dao DY, Hynan LS, Yuan HJ, et al. Two distinct subtypes of hepatitis $B$ virus-related acute liver failure are separable by quantitative serum immunoglobulin $\mathrm{M}$ anti-hepatitis B core antibody and hepatitis B virus DNA levels. Hepatology. 2012;55(3):676-684.

14. Krugman S, Overby LR, Mushahwar IK, et al. Viral hepatitis, type B. Studies on natural history and prevention re-examined. $N$ Engl J Med. 1979;300(3):101-106.

15. Maruyama T, Schodel F, Iino S, et al. Distinguishing between acute and symptomatic chronic Hepatitis B virus infection. Gastroenterology. 1994;106(4):1006-1015.

16. Rodella A, Galli C, Terlenghi L, et al. Quantitative analysis of HBsAg, IgM anti-HBc and anti-HBc avidity in acute and chronic hepatitis B. $J$ Clin Virol. 2006;37(3):206-212.

17. Gupta E, Bajpai M, Sharma P, Shah A, Sarin S. Unsafe injection practices: a potential weapon for the outbreak of blood borne viruses in the community. Ann Med Health Sci Res. 2013;2(3):177. 